\title{
Unveiling a new type of ultradense anomalous metallic glass with improved strength and ductility through a high-pressure heat treatment
}

\author{
Rui Yamada', Yuki Shibazaki $\mathbb{1}^{2}$, Yasuto Abe ${ }^{1}$, Wookha Ryu ${ }^{1}$ and Junji Saida (i]
}

\begin{abstract}
An anomalous glass was discovered through high-pressure heat treatment ( $5.5 \mathrm{GPa}$ at $850 \mathrm{~K}$ ) followed by rapid cooling of a $\mathrm{Zr}_{50} \mathrm{Cu}_{40} \mathrm{Al}_{10}$ metallic glass. Despite a reduction in the crystallization temperature and enthalpy, high-resolution transmission electron microscopy analysis revealed that the collected bulk sample maintained a fully amorphous structure. The density of the sample was $0.6 \%$ larger than that of the as-cast state and was even larger than that of the partially crystallized state. These results suggest the formation of an ultradense packing glass that cannot be obtained through conventional annealing. Compression test results indicated a significant increase in the Young's modulus and fracture strength, supporting the creation of an anomalous metallic glass. In addition, plasticity was observed in the treated sample. It was therefore concluded that the high-pressure heat treatment enabled the creation of a new type of glass that is normally overshadowed by the crystallized phase at atmospheric pressure. We explained the creation of the ultradense glass by introducing a pressure parameter $(P)$ to the conventional volume $(v)$ - temperature $(T)$ diagram.
\end{abstract}

\section{Introduction}

Recently, high-pressure treatment of metallic glasses has received significant scientific attention. In particular, polymorphic transition induced by pressure has been observed in many metallic glass systems ${ }^{1-5}$. The pressureinduced liquid-to-liquid transition was suggested by Dmowski et al. in Zr-based metallic glasses ${ }^{6}$. In molecular dynamic (MD) simulation studies, polymorphic transitions and the creation of new types of abnormal glass through high-pressure heat treatment was predicted (i.e., a high-energy but high-density and well-ordered glass $\left.{ }^{7,8}\right)$. Thus far, there have been attempts to explain these types of transitions and phenomena using many theoretical models (e.g., $4 \mathrm{f}$ electron delocalization ${ }^{1-5}$, P- and N-type free volume ${ }^{8,9}$, and a two-order-parameter $\left.\operatorname{model}^{10,11}\right)$. However, the detailed mechanisms remain unknown.

\footnotetext{
Correspondence: Rui Yamada (rui-yamada@fris.tohoku.ac.jp)

${ }^{1}$ Frontier Research Institute for Interdisciplinary Sciences (FRIS), Tohoku

University, Sendai 980-8578, Japan

${ }^{2}$ International Center for Young Scientists (ICYS), National Institute for Materials

Science (NIMS), Tsukuba 305-0044, Japan
}

Hence, high-pressure treatment research has mainly focused on clarifying these specific physical phenomena, leading to the appearance of several regulations regarding measurements. One is that most research has relied heavily on in situ X-ray diffraction measurements ${ }^{1-6}$. $\mathrm{X}$-ray diffraction measurements provide local average information, while the overall/bulk properties of the sample cannot be clarified using only this approach. Suzuki et al. previously reported that the mechanical behaviors of $\mathrm{Zr}_{50} \mathrm{Cu}_{40} \mathrm{Al}_{10}$ metallic glasses strongly depend on the observation scale (i.e., macroscopic and microscopic) ${ }^{12}$, which means that some of the properties (e.g., non-affine deformation) differ completely depending on the length scale of the target measurement. Therefore, microscopic and macroscopic measurements are important to clarify the overall properties of samples treated at high pressures.

Another regulation concerns the sample size. To find a significantly unique physical phenomenon (e.g., pressureinduced devitrification ${ }^{13}$ ), a high applied stress of several tens or even hundreds of gigapascals is required. As a 
result, the sample size has continuously decreased (e.g., the thickness is now several tens of micrometers), resulting in limited studies on the properties of the bulk samples treated at high pressures. All of these regulations hinder the clarification of the real phenomenon of the high-pressure experiment.

Previously, Dmowski et al. prepared a high-pressure heat-treated sample comprising a $\mathrm{Zr}$-based metallic glass and discussed the glasses created at the high pressures and high temperatures ${ }^{6}$. However, they investigated only the atomic-scale structures of the created glasses by using the X-ray diffraction technique so that the material properties (e.g., thermal and mechanical properties) of the sample were not evaluated. Therefore, the real state of the created glass through the treatment has yet to be understood.

The objective of the present study was to clarify the effect of high-pressure heat treatment on metallic glass by investigating the properties of bulk samples. We primarily focused on studying a high-pressure heat-treated bulk sample (i.e., diameter and height on the millimeter scale) by rapid cooling, with the aim of obtaining a sample frozen in the high-pressure/temperature glassy state at room temperature. Then, several types of mechanical and thermal measurements were performed to determine the glassy nature of the sample, and the anomalous features of the newly created metallic glass are discussed.

\section{Materials and methods Materials}

An alloy ingot with a nominal composition of $\mathrm{Zr}_{50} \mathrm{Cu}_{40} \mathrm{Al}_{10}$ (at.\%) was prepared by arc melting mixtures of pure metals in an argon atmosphere. Rod and disc samples with diameters of $4 \mathrm{~mm}$ and heights of 6-8 and $1 \mathrm{~mm}$, respectively, were prepared by cutting the tilt-cast samples. The amorphous nature was confirmed using Xray diffraction (XRD; Rigaku MultiFlex) and transmission electron microscopy (TEM; JEOL JEM-2000F) in advance. The thermal properties were measured using differential scanning calorimetry (DSC; TA Q-100) at a heating rate of $20 \mathrm{~K} / \mathrm{min}$.

\section{High-pressure heat treatment}

The high-pressure experiments were conducted on the rod samples using a belt-type large-volume press apparatus. In preparing the high-pressure heat-treated samples, we referred to a previous study by Dmowski et al. to determine the experimental conditions ${ }^{6}$. An annealing temperature of $850 \mathrm{~K}$, which might be slightly below $T_{x}$, and an applied pressure of $5.5 \mathrm{GPa}$ were selected. First, the quasi-isotropic pressure of $5.5 \mathrm{GPa}$ was applied to the sample at room temperature, and then, the system was heated to $850 \mathrm{~K}$. The heating rate was approximately $20 \mathrm{~K} / \mathrm{min}$ with a holding time of $2 \mathrm{~min}$ at $850 \mathrm{~K}$. The estimated cooling rate was as fast as $100 \mathrm{~K} / \mathrm{s}$, with the aim being to retain the high-pressure/temperature state at room temperature. After cooling the sample, the pressure was removed, and the high-pressure heat-treated bulk sample was collected.

\section{Reference samples}

The relaxed and partially and fully crystallized samples were prepared by annealing the as-cast glass at ambient pressure as references. The relaxed state was obtained by annealing the as-cast sample at $740 \mathrm{~K}\left(\sim 1.05 T_{g}\right)$ for $2 \mathrm{~min}$ followed by slow cooling $(20 \mathrm{~K} / \mathrm{min}$ cooling). The partially and fully crystallized states were prepared by annealing at $740 \mathrm{~K}\left(\sim 1.05 T_{g}\right)$ for $20 \mathrm{~min}$ and $873 \mathrm{~K}\left(\sim 1.24 T_{g}\right)$ for $1 \mathrm{~min}$, respectively.

Static annealing of the as-cast sample at ambient pressure Isothermal annealing of the as-cast sample at $740 \mathrm{~K}$ for $5,10,17,18,20,22$, and $23 \mathrm{~min}$ and successive isochronal DSC scans were conducted to evaluate the thermal properties/structural differences between the heat-treated sample under ambient pressure and the high-pressure heat-treated samples by comparing the crystallization temperature $\left(T_{x}\right)$ and crystallization enthalpy $\left(\Delta H_{\text {cry }}\right)$. The microstructures of the samples that were isothermally heat-treated at $740 \mathrm{~K}$ for 17,20 and 23 min were examined using TEM.

\section{Evaluation of bulk properties of high-pressure heat- treated sample}

The outer appearance of the collected bulk sample was examined using scanning electron microscopy (SEM; JEOL JSM-6010LV). DSC scans were performed using a heating rate of $20 \mathrm{~K} / \mathrm{min}$ to determine the thermal properties of the sample. Density measurements were performed using the rod samples with heights of $\sim 6 \mathrm{~mm}$ at room temperature using a gas pycnometer (Shimadzu Micromeritics AccuPycII 1340). The data were obtained by averaging 30 measurement points. The density measured using the Archimedes method was used as the standard data. The electric resistivity of each sample was measured using the four-terminal method. The measurement was performed five times, and the average was recorded. The amorphous nature was characterized using XRD and TEM. High-resolution transmission electron microscopy (HRTEM; FEI TITAN3 G2 60-300 Double Cs corrector) analysis was performed to investigate the internal microstructure at the atomic scale. The mechanical properties were evaluated using compression tests. The initial strain rate of $\varepsilon_{0} \sim 10^{-4} / \mathrm{s}$ was set during the test. The strain was measured with a strain gage (Kyowa Electronic Instruments, gage length of $1 \mathrm{~mm}$ ) attached on the surface of the sample. It was fixed at the center part using an instantaneous adhesive (Kyowa 
Electronic Instruments, cyanoacrylate base). More than three tests were conducted for each sample, and the results were obtained by averaging those data. The surface and side views of the fractured sample were observed using SEM (JEOL JSM-6010LV and FEI Versa 3D DualBeam). The surface and the shear band area on the side were cut using focused ion beam (FIB) milling (FEI Versa 3D DualBeam), and HRTEM was used to examine the microstructure.

\section{Results and discussion}

\section{Accessibility of high-pressure heat-treated state by} annealing as-cast glass at ambient pressure

DSC curves of the as-cast, relaxed, and high-pressure heat-treated $\mathrm{Zr}_{50} \mathrm{Cu}_{40} \mathrm{Al}_{10}$ samples are presented in Fig. 1 (a). The $T_{g}, T_{x}$, and $\Delta T_{x}$ of the as-cast sample were $704 \mathrm{~K}$, $786 \mathrm{~K}$, and $82 \mathrm{~K}$, respectively. For the relaxed sample, $T_{g}$ was determined to be $701 \mathrm{~K}$, which was almost the same as that of the as-cast state, whereas $T_{x}$ was slightly lower (781 K). During the annealing process, thermal accumulation (i.e., atomic ordering) may have occurred in the system ${ }^{14-16}$, resulting in the reduced crystallization temperature. Notably, for the high-pressure heat-treated sample, the glass transition temperature was almost the same $\left(T_{g}=703 \mathrm{~K}\right)$, whereas the crystallization temperature $\left(T_{x}=749 \mathrm{~K}\right)$ was lower than that of the as-cast state by $37 \mathrm{~K}$. In addition, the wide plateau region observed above $T_{g}$ in the as-cast and relaxed samples, which is considered to be a supercooled liquid state ${ }^{17}$, clearly diminished in the high-pressure heat-treated sample. These results suggest that the thermal stability decreased with the treatment.

As observed in Fig. 1a, the height of the crystallization peak for the high-pressure heat-treated sample also decreased compared with that of the other two curves. The crystallization enthalpies $\left(\Delta H_{c r y}\right)$ of the as-cast and relaxed samples were calculated to be 45.9 and $43.4 \mathrm{~J} / \mathrm{g}$, respectively. The enthalpy of the high-pressure heat-treated sample was significantly decreased $\left(\Delta H_{\text {cry, high pressure heat }}\right.$ treated $=25.4 \mathrm{~J} / \mathrm{g}$ ) and was only $55 \%$ of that of the as-cast state $\left(\Delta H_{\text {cry, as-cast }}=45.9 \mathrm{~J} / \mathrm{g}\right)$. For confirmation, we conducted DSC scans in the center and edge parts of the disk sample (see supplementary Fig. S1). The results revealed that the $T_{g}, T_{x}$ and $\Delta H_{c r y}$ results were almost the same in each part of the sample.

Based on the thermal analyses, one may suspect that the crystalline phase had already precipitated in the highpressure heat-treated sample. Figure $1 \mathrm{~b}$ shows the relationship between the crystallization temperature and enthalpy during isothermal annealing of the as-cast glass under ambient-pressure conditions. For a longer annealing time, a decreased crystallization temperature and enthalpy were observed. The results for the high-pressure heat-treated sample were also plotted, are shown in Fig. $1 \mathrm{~b}$, and are relatively close to those of the isothermally annealed samples for 22 and $23 \mathrm{~min}$. These results imply that similar thermal properties to those of the highpressure heat-treated state can be obtained even with static annealing at ambient pressure. However, the internal microstructure of the high-pressure heat-treated sample is significantly different from that of the isothermally annealed sample at ambient pressure. TEM analyses were conducted for the isothermally annealed (23 min) (not shown here) and high-pressure heat-treated samples [HRTEM data are shown in Fig. 2b]. The normal low-magnification TEM images and diffraction patterns (not shown here) revealed that the crystalline phase already precipitated in the matrix of the statically
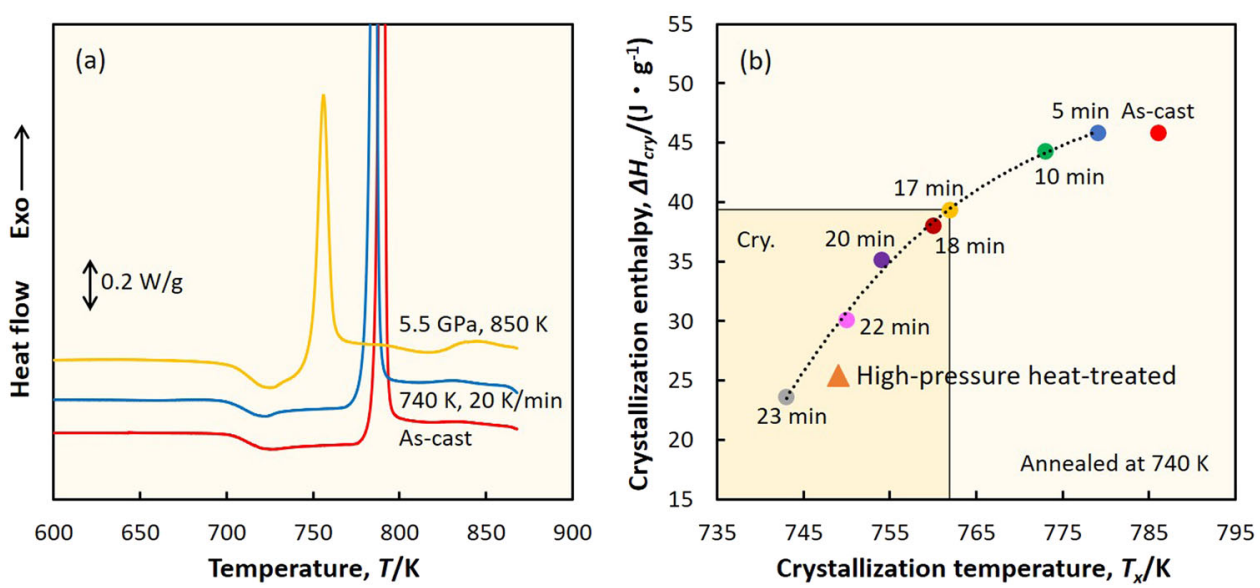

Fig. 1 a DSC curves of the as-cast, relaxed $(740 \mathrm{~K}, 20 \mathrm{~K} / \mathrm{min})$, and high-pressure heat-treated (5.5 GPa, $850 \mathrm{~K})$ samples of $\mathrm{Zr}_{50} \mathrm{Cu}_{40} \mathrm{Al}_{10}$ metallic glass. b Relationship between crystallization enthalpy $\Delta H_{c r y}$ and crystallization temperature $T_{x}$ of the as-cast and isothermally-annealed $(740 \mathrm{~K}$ for $5,10,17$, $18,20,22$, and $23 \mathrm{~min}$ ) samples. The triangle represents the data for the high-pressure heat-treated sample. The square area corresponds to the time beyond the incubation time for crystallization at $740 \mathrm{~K}$. 

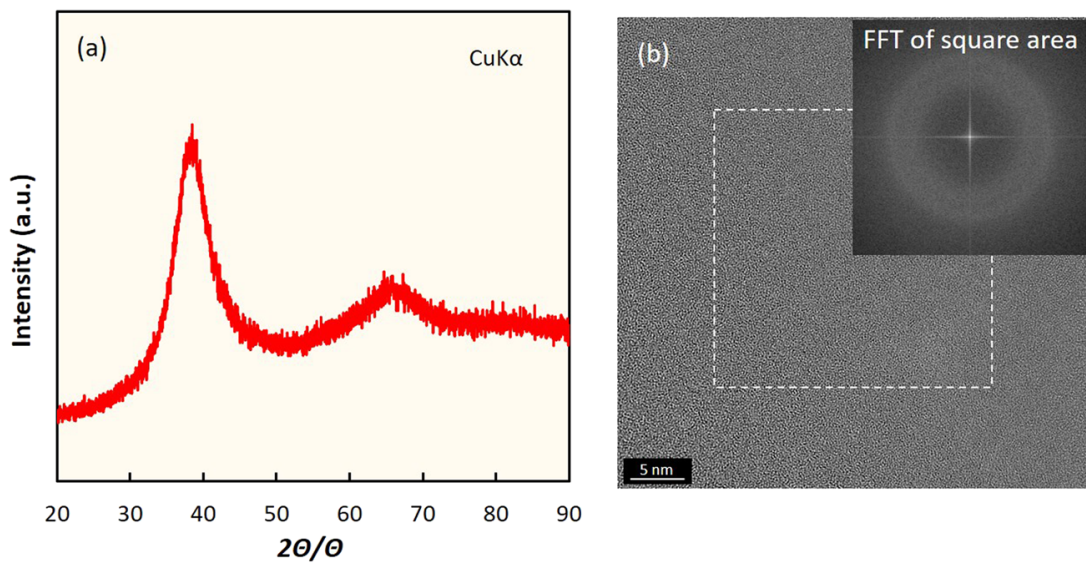

Fig. 2 a XRD pattern of the high-pressure heat-treated sample. $\mathbf{b}$ HRTEM image and FFT pattern of the treated sample.

annealed sample, whereas no contrast or clear diffraction spots were observed in the TEM images for the highpressure heat-treated sample. The XRD data of the cross section of the disk sample also indicated that the highpressure heat-treated sample maintained an amorphous structure (Fig. 2a). Furthermore, to investigate the possibility of small-scale crystallization (e.g., atomic ordering), which is hard to observe by the normal TEM observation, we performed HRTEM observations for the high-pressure heat-treated sample. Figure $2 b$ shows the HRTEM image and the fast Fourier transform (FFT) data from the center part of the disk sample. The typical salt-and-pepper-like pattern was confirmed in the image, and the FFT pattern contained only a diffuse halo ring, indicating a fully amorphous structure. Additionally, the synchrotron radiation X-ray diffraction data of the collected sample indicate an amorphous nature (see supplementary Fig. S2). Although the colored square area in Fig. 1b corresponds to the precipitation of a crystalline phase in the ambient-pressure annealed sample, the high-pressure heat-treated metallic glass contains no crystalline phase even in the colored square area and can be characterized as a fully amorphous structure at the atomic scale, indicating that such a unique state cannot be attained by annealing of the as-cast state at ambient pressure. In other words, under ambient-pressure conditions, crystallization inevitably occurs before attaining such a state during the annealing process.

\section{Unveiling the creation of an ultradense metallic glass thorough the measurement of bulk properties}

Figure 3 presents the density measurement results of the as-cast, relaxed, high-pressure heat-treated, and crystallized samples. The as-cast metallic glass had a density of $6.882 \mathrm{~g} / \mathrm{cm}^{3}$, which is almost the same as that reported elsewhere ${ }^{18}$. As expected, the density of the crystallized sample was the highest obtained herein at

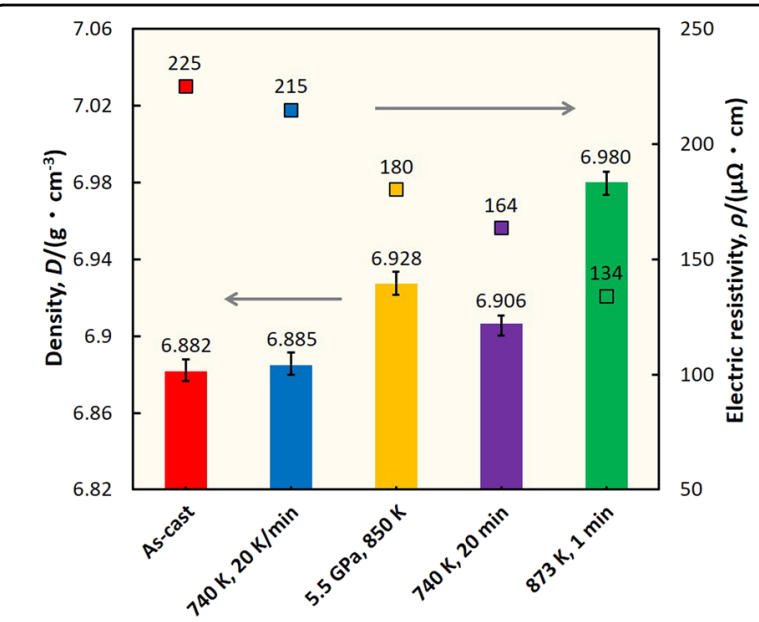

Fig. 3 Densities and electric resistivities of the as-cast, relaxed (740 K, $20 \mathrm{~K} / \mathrm{min})$, high-pressure heat-treated $(5.5 \mathrm{GPa}, 850 \mathrm{~K})$, partiallycrystallized (740 K, 20 min), and fully-crystallized (873 K, 1 min) samples.

$6.980 \mathrm{~g} / \mathrm{cm}^{3}$. When the glassy sample was heated above the crystallization temperature $\left(T_{x}\right)$, the atoms aligned periodically, and the glassy structure was transformed into a crystalline phase, resulting in a distinct volume shrinkage (increase in the density). The density of the highpressure heat-treated sample was $6.928 \mathrm{~g} / \mathrm{cm}^{3}$, which is $0.6 \%$ larger than that of the as-cast sample. It was expected that volume shrinkage of the high-pressure heattreated sample would occur because of the extremely close atomic distance under the high external pressure. However, it is important to note that the increase in the density through this treatment was extraordinarily high. For the relaxed state, the density was measured to be $6.885 \mathrm{~g} / \mathrm{cm}^{3}$, which is higher than that of the as-cast state; however, the increase was at most $0.05 \%$. The density increase in the high-pressure heat-treated sample was much higher than that for the relaxed state by roughly one 
order of magnitude. Yokoyama et al. previously reported the annealing time dependence of the density change of the same alloy system of $\mathrm{Zr}_{50} \mathrm{Cu}_{40} \mathrm{Al}_{10}$ metallic glass ${ }^{19,20}$. They changed the annealing time from 0 to $6.05 \times 10^{5} \mathrm{~s}$ ( 1 week) as well as the temperature $(473 \mathrm{~K}, 573 \mathrm{~K}$, and $673 \mathrm{~K}$, which are all below the $T_{g}$ ). The density change was dramatic at $673 \mathrm{~K}$ and almost saturated after the annealing time of $5.4 \mathrm{ks}$, which might suggest that the sample had already reached the metastable condition and that the free volume was almost annihilated. Nevertheless, the saturated value of the density was evaluated to be at most $0.4 \%$ higher than that of the as-cast state, but it was lower than the value of the density increases due to the highpressure heat treatment observed in this study (i.e., 0.6\%). Our results clearly indicate the creation of an ultradense random atomic configuration using the high-pressure heat treatment.

Figure 3 also shows the electric resistivities of each sample. The resistivity of the as-cast state was $225 \mu \Omega \mathrm{cm}$, and this value decreased to a slightly lower value of $215 \mu \Omega \mathrm{cm}$ with relaxation. Annihilation of the free volume resulted in a decreased atomic distance, leading to a more conductive state. In the crystallized state, the resistivity was lower $(134 \mu \Omega \mathrm{cm})$ than that of the amorphous states (i.e., the as-cast and relaxed states). As expected, the resistivity of the high-pressure heat-treated sample was much lower than those of the other amorphous states. This result was observed because the atomic distance decreased with high pressure, as easily predicted from the density measurement results, and caused a decrease in the electric resistivity. These electric resistivity results also support the creation of an ultradense glassy state.

Through microstructure observations and bulk property measurements, it was revealed that an anomalous glassy state with an abnormal random atomic configuration was constructed in the high-pressure heat-treated sample. In addition, such an unusual state cannot be obtained by simply annealing the as-cast sample at ambient pressure. Dmowski et al. investigated the change in the local atomic configuration at high pressures and high temperatures in the same $\mathrm{Zr}_{50} \mathrm{Cu}_{40} \mathrm{Al}_{10}$ alloy system ${ }^{6}$ as this study using a high-energy XRD technique and atomic pair distribution function (PDF) analysis and observed that the high pressure in the supercooled liquid region led to an increase in the atomic rearrangement, which agrees well with the present density measurement results. These authors also argued that the rearrangement appeared to be different from that achieved through a normal aging process. As we have already demonstrated, the high-pressure heat-treated state cannot be obtained through normal annealing at ambient pressure. Although the observed area and evaluation technique were completely different [in their case, the observable area was very small (i.e., the beam size was
$0.2 \mathrm{~mm} \times 0.2 \mathrm{~mm}$ ) and X-ray diffraction was used as the characterization method, whereas we investigated the density/electric resistivity for the collected bulk sample (i.e., a rod with a diameter of $4 \mathrm{~mm}$ and height of 6-8 $\mathrm{mm}$ )], our results are consistent with those in the previous report.

\section{Creation of a new type of hidden anomalous metallic glass}

As demonstrated above, the anomalous glass was created using a high-pressure heat treatment. To evaluate its anomalous features, a partially crystallized sample was also prepared by annealing the as-cast sample at $740 \mathrm{~K}$ for 20 min (i.e., longer than the incubation time for crystallization) as a reference. The crystalline volume fraction of the sample was estimated to be $V_{f}=\left\{1-\left(\Delta H_{\text {cry }, 20 \mathrm{~min}} /\right.\right.$ $\left.\left.\Delta H_{\text {cry,as }}\right)\right\} \times 100=23.5 \%$ from the result in Fig. 1(b). It was confirmed that the crystalline phase precipitated, and the sample was partially crystallized, by TEM image analysis (see supplementary Fig. S3). Surprisingly, the density of the partially crystallized sample was $6.906 \mathrm{~g} / \mathrm{cm}^{3}$, which is lower than that of the high-pressure heat-treated sample $\left(6.928 \mathrm{~g} / \mathrm{cm}^{3}\right)$ (see Fig. 3). Although the crystalline phase itself was much denser than that of the amorphous state, the remaining amorphous phase in the partially crystallized sample was thought to be not as dense. Therefore, the total density of the high-pressure heat-treated sample should be higher than that of the partially crystallized sample. Although a few studies have been reported ${ }^{21,22}$, it has been infrequently observed that a fully amorphous sample is denser than the already crystallized sample. The electrical resistivity measurement of the partially crystallized sample was also conducted, as shown in Fig. 3. The value resistivity was $164 \mu \Omega \mathrm{cm}$, which is lower than that of the high-pressure heat-treated sample. This is thought to be due to the substantially decreased electric resistivity of the precipitated crystalline phase than that of the amorphous matrix.

The above experimental results suggest the existence of a new type of glassy state, which is generally overshadowed by the crystallized state at ambient pressure. Here, we successfully demonstrate and identify a "hidden anomalous metallic glass" through bulk property measurements for the first time.

Figure 4a presents stress-strain curves of the different states under compression loading, and the Young's modulus and fracture strength determined from the compression tests are also presented in Fig. 4b. The detailed values of the mechanical properties are summarized in Table 1. For the as-cast state, the Young's modulus and fracture strength were measured to be $E_{\text {as-cast }}=96.0 \mathrm{GPa}$ and $\sigma_{f \text { as-cast }}=1735 \mathrm{MPa}$, respectively. When the sample was annealed at $740 \mathrm{~K}$ and relaxed at a cooling rate of $20 \mathrm{~K} / \mathrm{min}$, the values increased to $E_{\text {relax }}=$ $98.7 \mathrm{GPa}$ and $\sigma_{f}$, relax $=1904 \mathrm{MPa}$, respectively. For the 

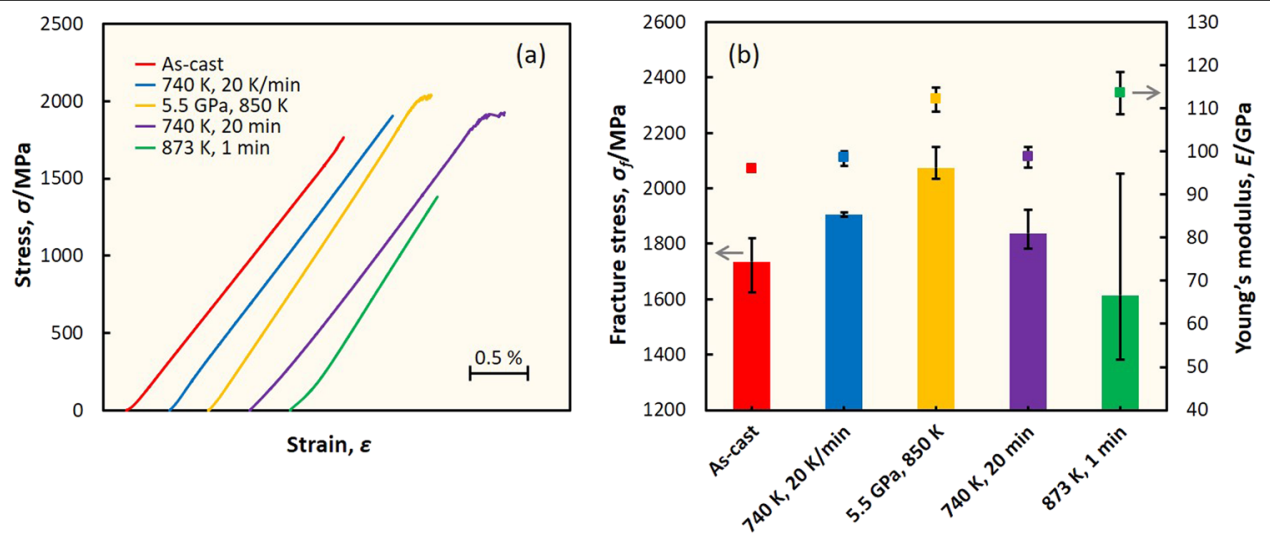

Fig. 4 a Stress-strain curves of various states obtained from compression tests. $\mathbf{b}$ Summary of results of fracture strength and Young's modulus of the samples.

Table 1 Mechanical properties of various samples obtained from compression tests.

\begin{tabular}{llll}
\hline & $\boldsymbol{E}(\mathrm{GPa})$ & $\boldsymbol{\sigma}_{\boldsymbol{f}}(\mathrm{MPa})$ & $\boldsymbol{\varepsilon}_{\boldsymbol{p}, \max }(\%)$ \\
\hline As-cast & 96.0 & 1735 & - \\
$740 \mathrm{~K}, 20 \mathrm{~K} / \mathrm{min}$ & 98.7 & 1904 & - \\
$5.5 \mathrm{GPa}, 850 \mathrm{~K}$ & 112.1 & 2074 & $\sim 0.15$ \\
$740 \mathrm{~K}, 20 \mathrm{~min}$ & 98.8 & 1838 & $\sim 0.30$ \\
$873 \mathrm{~K}, 1 \mathrm{~min}$ & 113.6 & 1615 & - \\
\hline
\end{tabular}

partially crystallized sample $(740 \mathrm{~K}, 20 \mathrm{~min})$, the Young's modulus and fracture strength values changed to $E_{\text {partially }}$ crystallize $=98.8 \mathrm{GPa}$ and $\sigma_{f}$, partially crystallize $=1838 \mathrm{MPa}$, respectively, which are similar to the results of the relaxed state. In contrast, a large difference is observed between the corresponding curves of the relaxed and partially crystallized samples in terms of plasticity. The partially crystallized sample exhibited plasticity $\left(\varepsilon_{p}, \max \sim 0.30\right)$ during the test, which resulted from pinning effects of the existing crystalline particle during compression. The crystalline particles prevented easy propagation of the shear bands and induced stress dispersion, leading to nucleation of multi shear bands and the appearance of considerable plasticity ${ }^{23-25}$. For the fully crystallized sample, the deviations in the Young's modulus and fracture strength were quite large. These deviations are thought to be attributed to the dependence of the direction between the compression load and orientation of the precipitates. Each sample had a different orientation of the crystals, which appeared to affect the results of the compression test. Interestingly, the Young's modulus of the high-pressure heat-treated sample reached $E_{\text {high pres- }}$ sure heat treated $=112.1 \mathrm{GPa}$, which was the largest among the other amorphous samples in this study and was almost the same as that of the fully crystallized sample $\left(E_{\text {fully crystallize }}=113.6 \mathrm{GPa}\right)$. Moreover, a maximum fracture strength of $\sigma_{f \text {, high pressure heat treated }}=2074 \mathrm{MPa}$ was observed for the high-pressure heat-treated alloy. This is recognized as an extraordinarily high strength, which indicates that the anomalous glass that was newly fabricated by the high-pressure heat treatment demonstrated an improvement in the mechanical properties.

It is also worth mentioning that the high-pressure heattreated sample obviously exhibited a certain plasticity of $\varepsilon_{p, \max } \sim 0.15 \%$ under a compressive load. The compression fracture of the treated sample exhibited a similar typical fracture geometry to that of the metallic glasses (see supplementary Fig. S4a), which indicates that the initial amorphous structure almost remained. A vein-like pattern was also observed in the fracture surface of the sample (Fig. 5c). It is noted that among the large shear bands, some were perpendicular to the fracture surface and the others were nearly parallel to the top and bottom surfaces in the high-pressure heat-treated sample (see supplementary Fig. S4a, b). This fracture morphology appears to be different from that of a typical metallic glass. The origin of the creation of the major shear bands is discussed later.

Miyazaki et al. indicated the achievement of a high fracture strength with plasticity through high-pressure heat treatment in a MD simulation ${ }^{7}$. The present results support this prediction experimentally. However, the origin of the plasticity in the high-pressure heat-treated sample remains unknown. We investigated the following two dominant factors.

One factor is the surface condition of the compression specimen. Huang et al. investigated the effect of shear predeformation on the mechanical performance of $\mathrm{Ti}$ based metallic glass ${ }^{26}$. They showed that the prefabrication of numerous shear bands on the surface of the sample can promote plastic strain during compression 


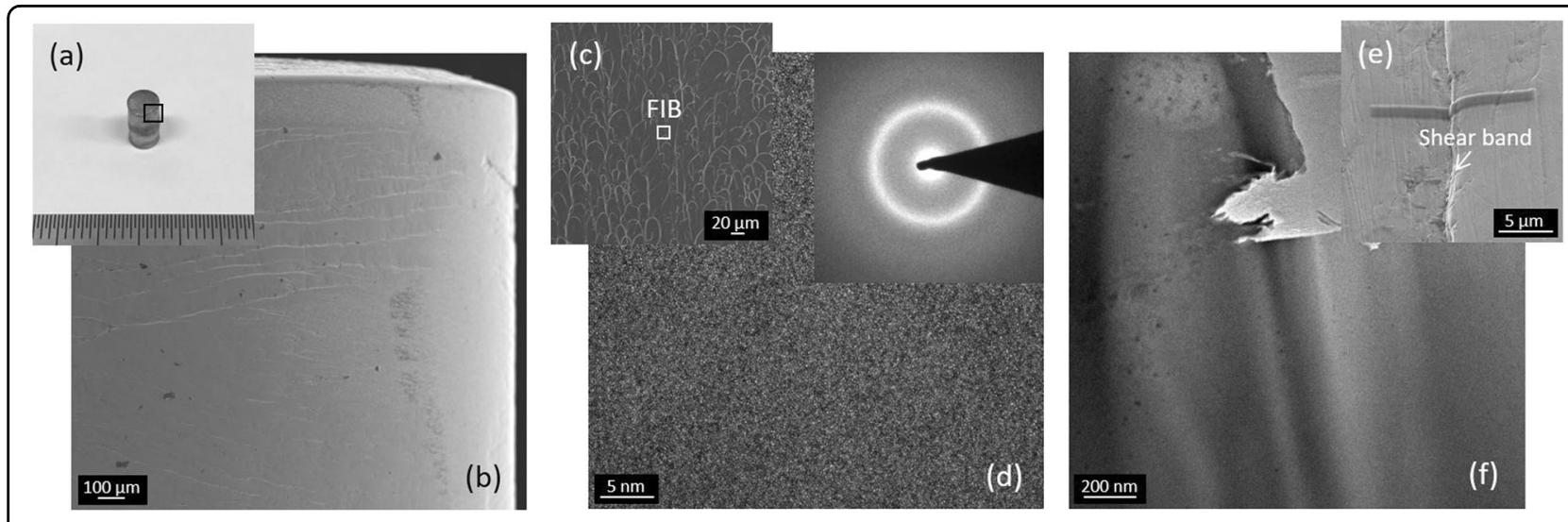

Fig. $\mathbf{5}$ a Outer appearance and $\mathbf{b}$ SEM image of the treated sample before compression test. c SEM and $\mathbf{d}$ HRTEM images of the fracture surface of the high-pressure heat-treated sample. e SEM and $\mathbf{f}$ TEM images of the shear band area of the fractured sample.

testing. Because the pressure condition in this study was quasi-hydrostatic, such an effect cannot be negligible. Figure $5 \mathrm{a}, \mathrm{b}$ shows the outer appearance of the highpressure heat-treated sample and SEM image of the surface of the sample before compression testing, respectively. As observed, many shear bands were prefabricated on the surface of the specimen. The observed large shear bands (see supplementary Fig. S4a and b) are thought to originate from one of them. It is possible that these shear bands, which were prefabricated in different directions, suppressed the growth and propagation of the initial bands. However, further investigation is needed to clarify the effect of the prefabricated shear bands on the improved plasticity.

The other related factor for the plasticity is deformation-induced crystallization during compression. As observed in the DSC curve (see Fig. 1a), the thermal stability of the high-pressure heat-treated sample was lower than that of the as-cast sample (e.g., the highpressure heat-treated sample exhibited a lower crystallization temperature than the as-cast one). Thus, it is possible that crystallization easily occurred during the compression test. In general, the strain energy induced by deformation complements the thermal energy for crystallization $^{27}$. It has also been demonstrated that deformation-induced crystallization promotes plasticity $^{28-30}$. To investigate the occurrence of deformationinduced crystallization during compression, the microstructure of the fracture surface was first examined using HRTEM, as shown in Fig. 5d. There is no obvious contrast or diffraction spots corresponding to the formation of a crystalline phase in the high-resolution image and diffraction pattern. On the other hand, crystalline particles were observed in the TEM image in the shear band area (Fig. 5e, f). Here, one would suspect that the crystalline phase had already precipitated in the prefabricated shear band area in the as-received high-pressure heat-treated sample. The TEM result (see supplementary Fig. S5) revealed that there were no crystalline regions in the area. These results indicate that deformation-induced crystallization surely occurred, which mainly led to the appearance of plasticity during the compression test.

The density and electric resistivity results (see Fig. 3) may indicate that a significantly relaxed state can be created inside the high-pressure heat-treated sample. We compared the mechanical properties of the corresponding sample with other alloy systems in the relaxed state. Figure $6 \mathrm{a}, \mathrm{b}$ presents the normalized Young's modulus $\left(E_{\text {relax }} / E_{\text {as-cast }}\right)$ and fracture strength $\left(\sigma_{f \text {, relax }} / \sigma_{f \text {, as-cast }}\right)$ of the relaxed state for several alloy systems reported in previous studies ${ }^{31-35}$. All the relaxed states were obtained through thermal annealing, and some of the samples contained small amounts of nanocrystalline/ordering. All of the alloys herein, including the present relaxed sample and except for the high-pressure heat-treated one, had ratios of less than 1.1. The obtained normalized ratios of the Young's modulus and fracture strength in the highpressure heat-treated state were $E_{\text {high pressure heat treated }} /$ $E_{\text {as-cast }}=1.17$ and $\sigma_{f \text {, high pressure heat treated }} / \sigma_{f \text {, as-cast }}=1.20$, respectively, which are far beyond 1.1. From these results, it is apparent that such high mechanical properties can only be obtained using the present high-pressure heat treatment and not through normal annealing. Notably, these values might be the highest values of those in any other reports for an almost fully amorphous structure.

Figure 7 shows a schematic illustration of how the ultradense glassy state was created through the highpressure heat treatment. We added a pressure axis to the conventional volume-temperature $(v-T)$ diagram, where an ambient pressure plane as well as a $5.5 \mathrm{GPa}$ plane exists. The $\mathrm{a}-\mathrm{a}^{\prime}$ line represents the liquid equilibrium line at ambient pressure, while $\mathrm{b}^{-\mathrm{b}^{\prime}}$ is the line at $5.5 \mathrm{GPa}$ (the gradation area $a-a^{\prime}-b-b^{\prime}$ corresponds to the liquid equilibrium plane). When a pressure is applied, the glass first 
follows the $\mathrm{A} \rightarrow \mathrm{B}$ path (i.e., transition from the ambient pressure plane to the $5.5 \mathrm{GPa}$ plane). Then, during heating with pressure ( $\mathrm{B} \rightarrow \mathrm{C}$ path), the glass tends to follow the liquid equilibrium line at $5.5 \mathrm{GPa}\left(\mathrm{b}-\mathrm{b}^{\prime}\right)$. It is noted that whether or not the glass overshoots the $b-b^{\prime}$ line is still uncertain (i.e., if overshoots, it suggests the presence of glass transition in the high-pressure conditions. However, whether or not the glass transition occurs is still unknown). During the cooling process, the sample follows the $\mathrm{C} \rightarrow \mathrm{D}$ path (still on the $5.5 \mathrm{GPa}$ plane). After removing the pressure ( $D \rightarrow E$ path), even though the elastic part should be

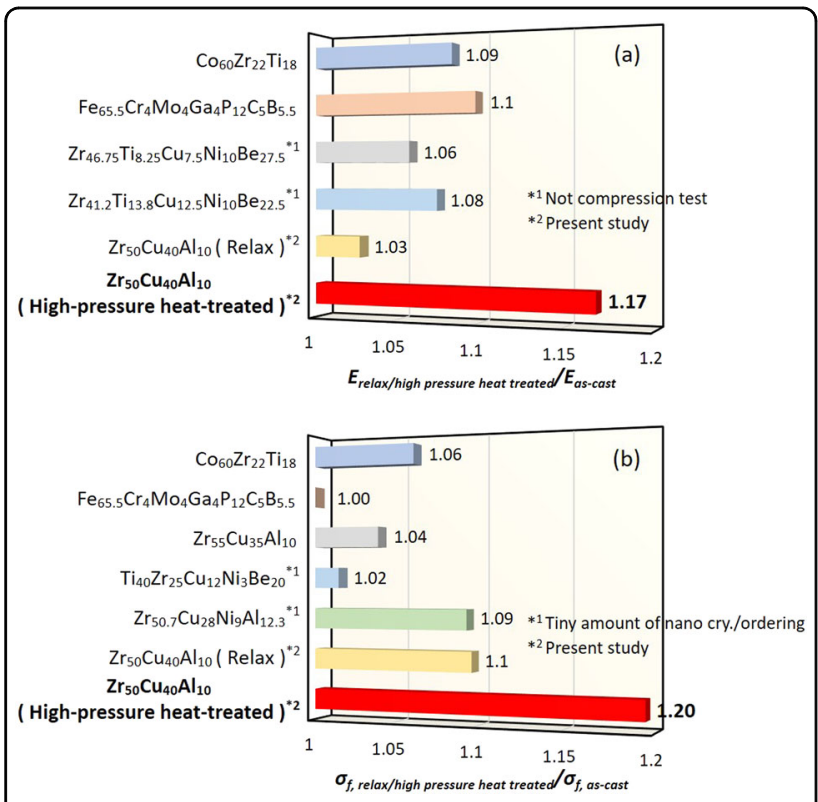

Fig. 6 a Normalized Young's modulus and $\mathbf{b}$ fracture strength of relaxed samples obtained from previous reports. The data for the relax and high-pressure heat-treated sample obtained in the present study are also provided. recovered, the glass may almost keep its state owing to the quenching (i.e., a glassy state at $5.5 \mathrm{GPa}$ is created that is mostly frozen in even after removing the pressure). The volume of the high-pressure heat-treated sample $\left(v_{1}\right)$ is much smaller than that in the initial state at ambient pressure $\left(v_{0}\right)$, namely, $v_{0} \gg v_{1}$. Regarding the experimental time scale (as already mentioned above by comparing the density data with the result by Yokoyama ${ }^{19,20}$ ), such an anomalous state seems to be difficult to attain only considering the ambient pressure plane. Adding the pressure axis in the $v-T$ diagram enables the creation of an ultradense state, which is not accessible by annealing the metallic glass at ambient pressure only.

Previous reports have claimed the creation of a wellordered local structure but a glass in a high potential energy state was revealed by a high-pressure heat treatment in simulation $^{7,8}$. Thus far, it has been difficult to discuss the real glassy nature of the high-pressure heat-treated alloy. It was experimentally confirmed in Fig. 3 that the glass exhibited ultradense packing, which implies that a wellordered glass might be created. However, details, such as whether or not the volume of the high-pressure heat-treated state can be below the liquid equilibrium line (i.e., whether or not the point $\mathrm{E}$ in Fig. 7 should be placed below a-a' line) and the energy state of the sample, remain uncertain. Further studies are required to determine the glassy state (e.g., the relationship between the energy and volume state) as well as the local atomic configuration to clarify the actual nature of the high-pressure heat-treated glass.

\section{Conclusions}

The high-pressure heat-treated $(5.5 \mathrm{GPa}$ at $850 \mathrm{~K}$ ) state of a $\mathrm{Zr}_{50} \mathrm{Cu}_{40} \mathrm{Al}_{10}$ metallic glass was successfully obtained by rapidly cooling a bulk sample (size of a few millimeters) from slightly below $T_{x}$. The obtained thermal properties
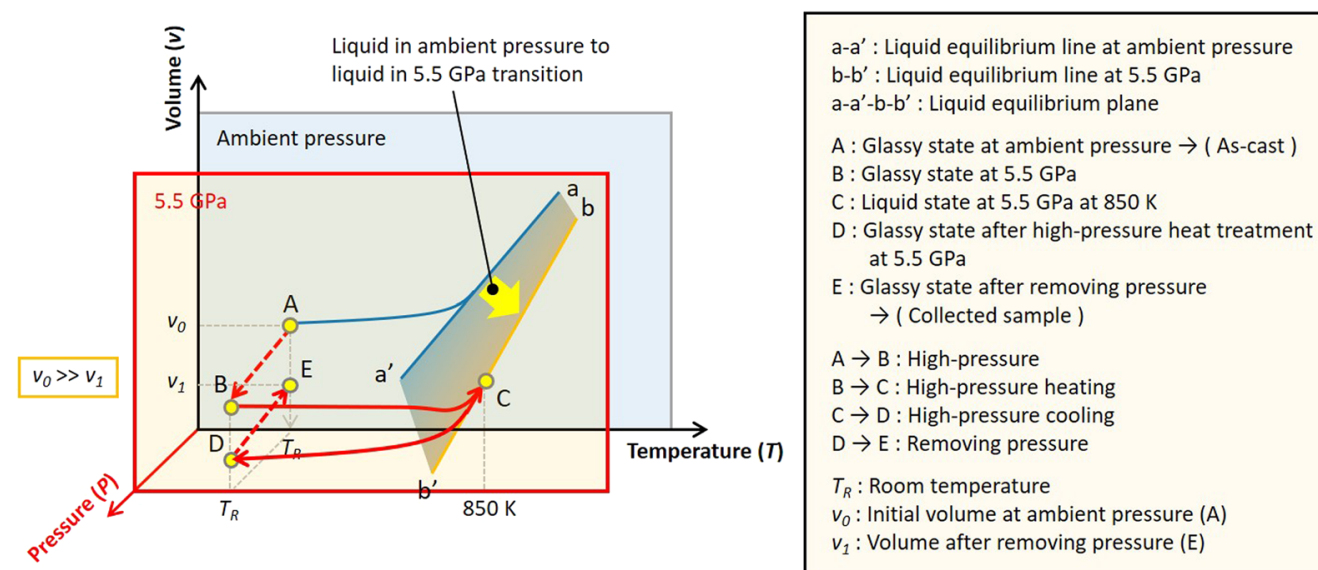

Fig. 7 Schematic illustration of volume $(v)$-Pressure $(P)$-Temperature $(T)$ diagram of the metallic glass. The process of creating ultradense glassy state through the high-pressure heat treatment is described $(A \rightarrow B \rightarrow C \rightarrow D \rightarrow E)$. 
were similar to those of the partially crystallized sample prepared by annealing the as-cast glass alloy at ambient pressure. Nevertheless, the microstructure of the highpressure heat-treated sample confirmed that a fully amorphous structure was maintained. The density and electric resistivity measurements revealed the creation of an ultradense packed metallic glass. In particular, the density was larger than that of the partially crystallized state, indicating that such a state is not readily accessible under ambient conditions. The significantly relaxed state might have been fabricated by the present high-pressure treatment; however, further investigations are needed to clarify the actual nature of the glass. It was confirmed that the high-pressure heat-treated sample exhibited excellent mechanical properties compared with those of other previously reported relaxed metallic glasses. A new concept, namely, a pressure factor $(P)$, was added to the volume $(v)$ - temperature $(T)$ diagram to account for the creation of an ultradense metallic glass. We successfully revealed a "hidden anomalous glassy state" and created a new type of metallic glass for the first time using a highpressure heat treatment process.

\section{Acknowledgements \\ This work was supported by a Grant-in-Aid from the Ministry of Education, Sports, Culture, Science and Technology, Japan, Scientific Research (A) (No. 18H03829) and by the "Promoted Program for Interdisciplinary Research" of the Frontier Research Institute for Interdisciplinary Sciences (FRIS), Tohoku University. The authors would also like to thank Tiffany Jain, M.S., from Edanz Group (www.edanzediting.com/ac) for editing a draft of this manuscript.}

\section{Conflict of interest}

The authors declare that they have no conflict of interest.

\section{Publisher's note}

Springer Nature remains neutral with regard to jurisdictional claims in published maps and institutional affiliations.

Supplementary information is available for this paper at https://doi.org/ 10.1038/s41427-019-0175-1.

Received: 22 April 2019 Revised: 18 September 2019 Accepted: 19 September 2019.

Published online: 6 December 2019

\section{References}

1. Sheng, H. W. et al. Polyamorphism in a metallic glass. Nat. Mater. 6, 192-197 (2007).

2. Zhen, Q. S. et al. Origin of pressure-induced polyamorphism in Ce75Al25 metallic glass. Phys. Rev. Lett. 104, 105702 (2010).

3. Lou, H. B. et al. Pressure-induced amorphous-to-amorphous configuration change in Ca-Al metallic glasses. Sci. Rep. 2, 376 (2012).

4. Zeng, Q. et al. General 2.5 power law of metallic glasses. Proc. Natl Acad. Sci. USA 113, 1714-1718 (2016).

5. Li, L. et al. Polyamorphism in Yb-based metallic glass induced by pressure. Sci. Rep. 7, 46762 (2017).

6. Dmowski, W. et al. Pressure induced liquid to liquid transition in Zr-based supercooled melts and pressure quenched glasses. Sci. Rep. 7, 6564 (2017).

7. Miyazaki, N., Wakeda, M., Wang, Y. J. \& Ogata, S. Prediction of pressurepromoted thermal rejuvenation in metallic glasses. npj Computational Mater. 2, 16013 (2016)
8. Miyazaki, N., Lo, Y. C., Wakeda, M. \& Ogata, S. Properties of high-density, wellordered, and high-energy metallic glass phase designed by pressurized quenching. Appl. Phys. Lett. 109, 091906 (2016).

9. Egami, T. Structural relaxation in metallic glasses. Ann. NY. Acad. Sci. 371, 238-251 (1981).

10. Ding, J., Asta, M. \& Ritchie, R. O. Anomalous structure-property relationships in metallic glasses through pressure-mediated glass formation. Phy. Rev. B 93, 140204 (2016).

11. Tanaka, H. Two-order-parameter model of the liquid-glass transition. I. Relation between glass transition and crystallization. J. Non-Cryst. Solids 351, 3371-3384 (2005).

12. Suzuki, $\mathrm{H}$. et al. Investigation of elastic deformation mechanism in as-cast and annealed eutectic and hypoeutectic Zr-Cu-Al metallic glasses by multiscale strain analysis. Metals 6, 12 (2016).

13. Zeng, Q. et al. Long-range topological order in metallic glass. Science $\mathbf{3 3 2}$, 1404-1406 (2011).

14. Yamada, R., Tanaka, N., Guo, W. \& Saida, J. Crystallization behavior of thermally rejuvenated Zr50Cu40Al10 metallic glass. Mater. Trans. 58, 1463-1468 (2017).

15. Guo, W., Yamada, R., Saida, J., Lü, S. \& Wu, S. Thermal rejuvenation of a heterogeneous metallic glass. J. Non-Cryst. Solids 498, 8-13 (2018).

16. Guo, W., Saida, J., Zhao, M., Lü, S. \& Wu, S. Thermal rejuvenation of an Mgbased metallic glass. Metal. Mater. Trans. A 50, 1125-1129 (2019).

17. Gulzar, A. et al. Correlation between flow units and crystallization in metallic glasses. J. Non-Cryst. Solids 461, 61-66 (2017).

18. Haruyama, O., Yokoyama, Y. \& Inoue, A. Precise measurement of density in the isothermal relaxation processes of Pd42.5Cu30Ni7.5P20 and Zr50Cu40Al10 glasses. Mater. Trans. 48, 1708-1710 (2007).

19. Yokoyama, Y. et al. Evolution of mechanical properties of cast Zr50Cu40Al10 glassy alloys by structural relaxation. Mater. Trans. 46, 2755-2761 (2005).

20. Yokoyama, Y., Yamasaki, T., Liaw, P. K. \& Inoue, A. Study of the structural relaxation-induced embrittlement of hypoeutectic Zr-Cu-Al ternary bulk glassy alloys. Acta Mater. 56, 6097-6108 (2008).

21. Shen, T. D., Harms, U. \& Schwarz, R. B. Correlation between the volume change during crystallization and the thermal stability of supercooled liquids. Appl. Phys. Lett. 83, 4512 (2003).

22. $\mathrm{Hu}, \mathrm{Q}$. et al. Anomalous thermal expansion in the deep supercooled liquid region of a ZrCuAlAg bulk metallic glass. Mater. Res. Lett. 6, 121-129 (2018).

23. Lee, S. W., Huh, M. Y., Fleury, E. \& Lee, J. C. Crystallization-induced plasticity of Cu-Zr containing bulk amorphous alloys. Acta Mater. 54, 349-355 (2006).

24. Hajlaoui, K. et al. Plasticity induced by nanoparticle dispersions in bulk metallic glasses. J. Non-Cryst. Solids 353, 327-331 (2007).

25. Inoue, A., Fan, C., Saida, J. \& Zhang, T. High-strength Zr-based bulk amorphous alloys containing nanocrystalline and nanoquasicrystalline particles. Sci. Technol. Adv. Mater. 1, 73-86 (2000).

26. Huang, Y., Sun, Y. \& Shen, J. Tuning the mechanical performance of a Ti-based bulk metallic glass by pre-deformation. Intermetallics 18, 2044-2055 (2010).

27. Sun, J. F., Huang, Y. J., Shen, J., Wang, G. \& McCartney, D. G. Superplastic formability of a Zr-Ti-Ni-Cu-Be bulk metallic glass. J. Alloy. Compd. 415, 198-203 (2006)

28. Kumar, G., Ohkubo, T., Mukai, T. \& Hono, K. Plasticity and microstructure of ZrCu-Al bulk metallic glasses. Scr. Mater. 57, 173-176 (2007).

29. Saida, J., Setyawan, A. D., Kato, H. \& Inoue, A. Nanoscale multistep shear band formation by deformation-induced nanocrystallization in Zr-Al-Ni-Pd bulk metallic glass. Appl. Phys. Lett. 87, 151907 (2005).

30. Setyawan, A. D., Saida, J., Kato, H., Matsushita, M. \& Inoue, A. Deformationinduced structural transformation leading to compressive plasticity in Zr65Al7.5Ni10Cu12.5M5 ( $\mathrm{M}=\mathrm{Nb}, \mathrm{Pd})$ glassy alloys. J. Mater. Res. 25, 1149-1158 (2010)

31. Concustell, A. et al. Effect of relaxation and primary nanocrystallization on the mechanical properties of Cu60Zr22Ti18 bulk metallic glass. Intermetallics 13, 1214-1219 (2005).

32. Stoica, M. et al. Mechanical behavior of Fe65.5Cr4Mo4Ga4P12C5B5.5 bulk metallic glass. Intermetallics 13, 764-769 (2005).

33. Kumar, G., Rector, D., Conner, R. D. \& Schroers, J. Embrittlement of Zr-based bulk metallic glasses. Acta Mater. 57, 3572-3583 (2009).

34. Ohkubo, T., Nagahama, D., Mukai, T. \& Hono, K. Stress-strain behavior of Ti-based bulk metallic glass and their nanostructures. J. Mater. Res. 22, 1406-1413 (2007).

35. Huang, $Y$. et al. The effects of annealing on the microstructure and the dynamic mechanical strength of a ZrCuNiAl bulk metallic glass. Intermetallics 42, 192-197 (2013). 